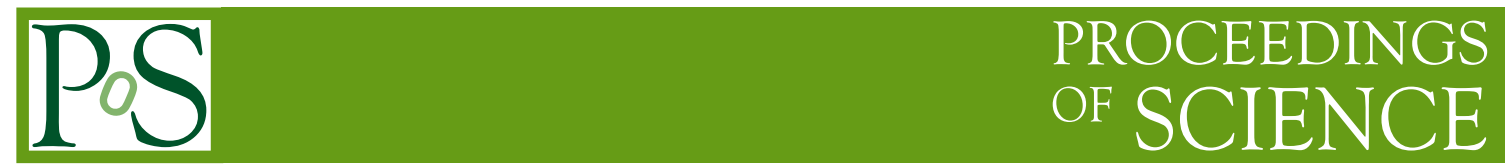

\title{
Heavy flavor results from CMS
}

\author{
P. Ronchese on behalf of the CMS collaboration* \\ Dipartimento di Fisica e Astronomia \\ Università di Padova and INFN, I-35131 Padova, ITALY \\ E-mail: paolo.roncheseepd.infn.it
}

Heavy flavor particles produced in LHC $p p$ collisions at $\sqrt{s}=7,8$ and $13 \mathrm{TeV}$ constitute an excellent opportunity to test the Standard Model and probe for new physics effects. Recent results by the CMS Collaboration on heavy flavor production and decays are presented.

XXV International Workshop on Deep-Inelastic Scattering and Related Subjects 3-7 April 2017

University of Birmingham, $U K$

${ }^{*}$ Speaker. 


\section{Introduction}

There are several motivations to study heavy flavour physics at CMS; they include the aim to probe the underlying QCD processes, and that's obtained measuring production cross-section, measuring polarizations and looking for new and exotic states. Another motivation is to look for effects of new physics beyond the Standard Model through the study of lifetime and decay properties of $\mathrm{B}$ hadrons, especially in rare decays.

\section{Data samples and selections}

Some results obtained from the analysis of data collected by CMS [1] in 2012 at $\sqrt{s}=8 \mathrm{TeV}$ and 2015 at $\sqrt{s}=13 \mathrm{TeV}$, corresponding to an integrated luminosity $\mathscr{L} \sim 20 \mathrm{fb}^{-1}$ and $\mathscr{L} \sim$ $2.7 \mathrm{fb}^{-1}$ respectively, will be shown in the following.

Dedicated triggers have been developed for the analyses to achieve a sustainable trigger rate when collecting data at the very high luminosities provided by LHC. The presence of two muons was required, forming a secondary vertex displaced from the primary interaction point and a momentum direction compatible with the flight direction.

\section{3. $B^{+}$production cross-section}

One result obtained from data collected at $\sqrt{s}=13 \mathrm{TeV}$ is the measurement of the $B^{+}$differential production cross-section. The cross-section has been measured in the decay channel $B^{ \pm} \rightarrow J / \psi K^{ \pm}, J / \psi \rightarrow \mu^{+} \mu^{-}$in bins of transverse momentum up to $100 \mathrm{GeV}$, and rapidity up to 2.1 as shown in Fig.1 [2]. The results have been compared with corresponding ones obtained at $\sqrt{s}=7 \mathrm{TeV}[3]$ and predictions.
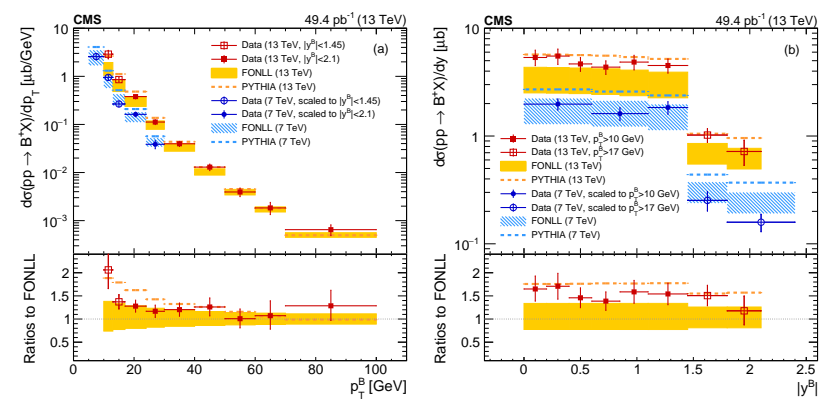

Figure 1: Differential cross-section for $B^{+}$production vs. transverse momentum (left) and rapidity (right) [2, 3], compared with PYTHIA simulation [4] and FONLL predictions [5, 6].

\section{Quarkonia production cross-section}

Another result obtained from data collected at $\sqrt{s}=13 \mathrm{TeV}$ is the measurement of quarkonia production cross-section, that can be used to test non-relativistic QCD and factorization models.

In those models quarkonia production is described as a two phases process where a perturbative production of a $q \bar{q}$ pair, in a singlet or octet state [7], is followed by hadronization described by 
long-distance matrix elements [8]. The measurement of the cross-section at different collision energies allows a test of this model: perturbative calculations can be done according to collision energy, while the same matrix elements can be used for all energies.

In Fig. 2 the cross section versus transverse momentum is shown, for charmonia and bottomonia [9], together with the cross-section measured at $\sqrt{s}=7 \mathrm{TeV}[10,11]$.
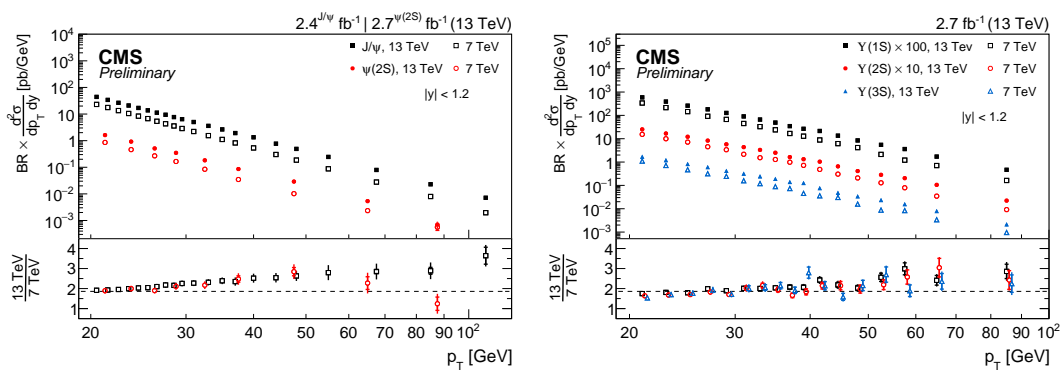

Figure 2: Differential cross-section for charmonia (left) and bottomonia (right) production vs. transverse momentum [9-11].

For charmonia an additional non-prompt component originating from the decay of $b$-hadrons must be taken into account. The prompt and non-prompt yields have been measured by simultaneously fitting the mass and the pseudo-proper decay length distributions. In Fig. 3 the $J / \psi$ and $\psi(2 \mathrm{~S})$ non-prompt fractions are shown versus the transverse momentum.
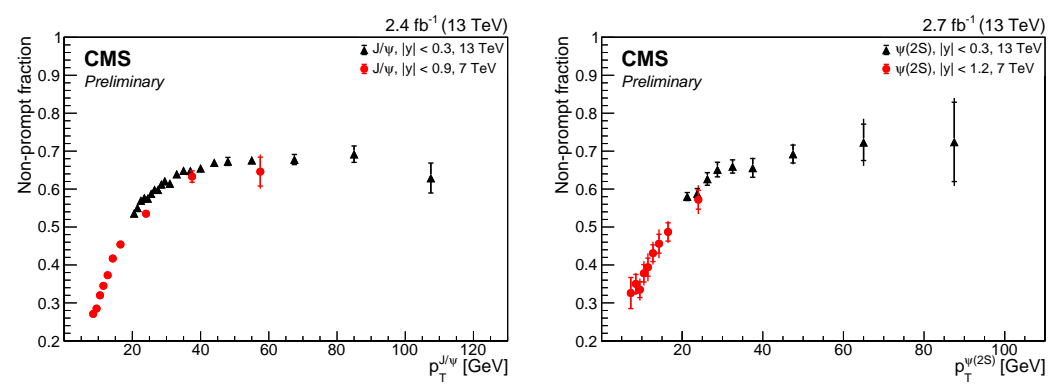

Figure 3: Non-prompt fraction for $J / \psi$ (left) and $\psi(2 \mathrm{~S})$ (right) vs. transverse momentum [9].

\section{5. $B^{0} \rightarrow K^{* 0} \mu^{+} \mu^{-}, K^{* 0} \rightarrow K^{+} \pi^{-}$angular analysis}

The decay $B^{0} \rightarrow K^{* 0} \mu^{+} \mu^{-}$is described by loop diagrams where the presence of new particles could give visible effects, specifically through contributions to Wilson coefficient $C_{7,9,10}$. Such effects have been tested performing an angular analysis and looking at the decay rate depending on the decay angles, $\theta_{l}, \theta_{K}, \phi$ defined as shown in Fig.4, and the dimuon invariant mass squared $q^{2}$.

The decay rate dependence on those variables is described by a set of parameters, including the muon forward/backward asymmetry $A_{\mathrm{FB}}$, the $K^{* 0}$ longitudinal polarization $F_{\mathrm{L}}$, the $K^{+} \pi^{-} S$-wave contribution $F_{\mathrm{S}}$ and the interference $A_{\mathrm{S}}$ betwen the $S$-wave and $P$-wave decays. Three more parameters, $P_{1}, P_{5}^{\prime}, A_{5 S}$, are present only in the terms depending on the angle $\phi$; they were first measured by the LHCb experiment [12], which observed a discrepancy of $P_{5}^{\prime}$ versus the Standard Model predictions. That discrepancy could be a hint of new physics effects [13], but there's also a claim that 


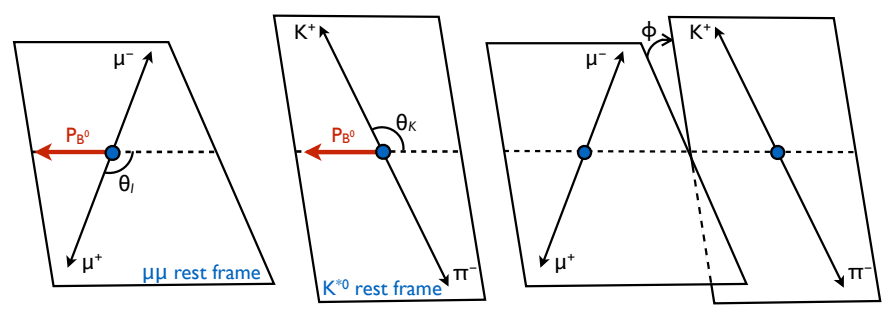

Figure 4: Sketch showing the definition of the angular observables $\theta_{l}$ (left), $\theta_{K}$ (middle), $\phi$ (right) for the decay $B^{0} \rightarrow K^{* 0} \mu^{+} \mu^{-}, K^{* 0} \rightarrow K^{+} \pi^{-}$.

it's possible to account for that effect in the Standard Model by refitting all loop contributions, with a special care for charm effects $[14,15]$.

The angular analysis has been performed in two phases; in the first phase the $\phi$ dependence has been integrated out, so that the parameters $P_{1}, P_{5}^{\prime}, A_{5 S}$ disappeared from the PDF:

$$
\begin{aligned}
\frac{1}{\Gamma} \frac{\mathrm{d}^{3} \Gamma}{\mathrm{d} \cos \theta_{K} \mathrm{~d} \cos \theta_{l} \mathrm{~d} q^{2}}= & \frac{9}{16}\left\{\frac{2}{3}\left[F_{S}+A_{S} \cos \theta_{K}\right]\left(1-\cos ^{2} \theta_{l}\right)\right. \\
+\left(1-F_{S}\right) & {\left[2 F_{L} \cos ^{2} \theta_{K}\left(1-\cos ^{2} \theta_{l}\right)\right.} \\
& +\frac{1}{2}\left(1-F_{L}\right)\left(1-\cos ^{2} \theta_{K}\right)\left(1+\cos ^{2} \theta_{l}\right) \\
& \left.\left.+\frac{4}{3} A_{F B}\left(1-\cos ^{2} \theta_{K}\right) \cos \theta_{l}\right]\right\}
\end{aligned}
$$

Decays of $B^{0} / \bar{B}^{0}$ were discriminated according to $K, \pi$ charges, where particle masses were assigned on $K^{* 0}$ candidate invariant mass basis. The angular distribution was fitted in $q^{2}$ bins with a PDF having 3 components for correctly tagged signal, mistagged signal, i.e. events where kaon and pion charges are misassigned, and background. The fitted variables are the invariant mass and the $\theta_{l, K}$ angles; the mass parameters, the yields and the angular parameters for the signal were left free; the angular parameters for the background have been fitted with sidebands and then kept fixed. The differential branching fraction is computed from a comparison with the corresponding branching ratio with a resonant dimuon system in the final state:

$$
\frac{\mathrm{d} \mathscr{B}\left(B^{0} \rightarrow K^{* 0} \mu^{+} \mu^{-}\right)}{\mathrm{d} q^{2}}=\frac{Y_{S}}{Y_{N}} \frac{\varepsilon_{N}}{\varepsilon_{S}} \frac{\mathscr{B}\left(B^{0} \rightarrow J / \psi K^{* 0}\right)}{\Delta q^{2}}
$$

where $Y_{S, N}$ are the event yields for the signal and normalization channel and $\varepsilon_{S, N}$ are the corresponding selection efficiencies.

Results obtained with data collected at $\sqrt{s}=8 \mathrm{TeV}$ are shown in Fig.5 [16]; Standard Model predictions [17-19] are compatible with CMS results.

In the second phase of the analysis the dependence on the angle $\phi$ has been included back, but due to the limited number of events to allow a fit a folding was applied; the angle $\phi$ itself was folded around 0 and $\theta_{l}$ was folded around $\pi / 2$. The fitted PDF was then:

$$
\begin{aligned}
& \frac{1}{\mathrm{~d} \Gamma / \mathrm{d} q^{2}} \frac{\mathrm{d}^{4} \Gamma}{\mathrm{d} \cos \theta_{K} \mathrm{~d} \cos \theta_{l} \mathrm{~d} \phi \mathrm{d} q^{2}}=\frac{9}{8 \pi}\left\{\frac { 2 } { 3 } \left[\left(F_{S}+A_{S} \cos \theta_{K}\right)\left(1-\cos ^{2} \theta_{l}\right)\right.\right. \\
& \left.+A_{5 S} \sqrt{1-\cos ^{2} \theta_{K}} \sqrt{1-\cos ^{2} \theta_{l}} \cos \phi\right] \\
& +\left(1-F_{S}\right)\left[2 F_{L} \cos ^{2} \theta_{K}\left(1-\cos ^{2} \theta_{l}\right)\right. \\
& +\frac{1}{2}\left(1-F_{L}\right)\left(1-\cos ^{2} \theta_{K}\right)\left(1+\cos ^{2} \theta_{l}\right) \\
& +\frac{1}{2} P_{1}\left(1-F_{L}\right)\left(1-\cos ^{2} \theta_{K}\right)\left(1-\cos ^{2} \theta_{l}\right) \cos 2 \phi \\
& \left.\left.+2 P_{5}^{\prime} \cos \theta_{K} \sqrt{F_{L}\left(1-F_{L}\right)} \sqrt{1-\cos ^{2} \theta_{K}} \sqrt{1-\cos ^{2} \theta_{l}} \cos \phi\right]\right\}
\end{aligned}
$$



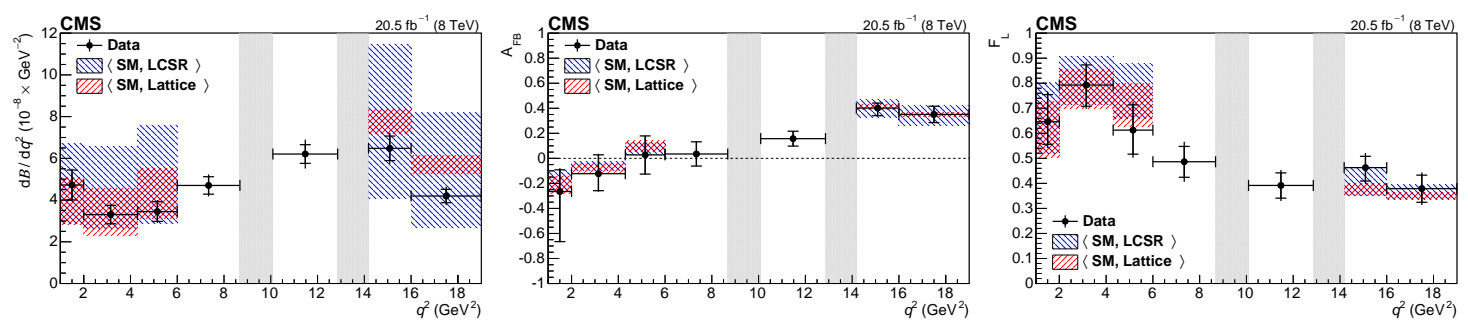

Figure 5: Measured values of $\mathrm{d} \mathscr{B} / \mathrm{d} q^{2}$ (left), $A_{\mathrm{FB}}$ (middle) and $F_{\mathrm{L}}$ (right) versus $q^{2}$ for $B^{0} \rightarrow K^{* 0} \mu^{+} \mu^{-}$[16], compared with the SM predictions obtained from light-cone sum rules (blue) $[17,18]$ and lattice QCD (red) [19].

The parameters $F_{L}, F_{S}$ and $A_{S}$ were fixed to the results obtained in the previous analysis and the consequences of this choice have been accounted for in the systematic error. A blind procedure was used, fitting signal in simulation only and using data to fit a control region before applying the procedure to data signal. The requirement of having a non-negative PDF sets boundaries to the parameters space; as a consequence the statistical error determination was not trivial, and a two-dimensional Feldman-Cousins scan was used, leaving $A_{5 S}$ as a nuisance parameter.

Results obtained with data collected at $\sqrt{s}=8 \mathrm{TeV}$ are shown in Fig.6 [21], compared with results from other experiments and predictions.
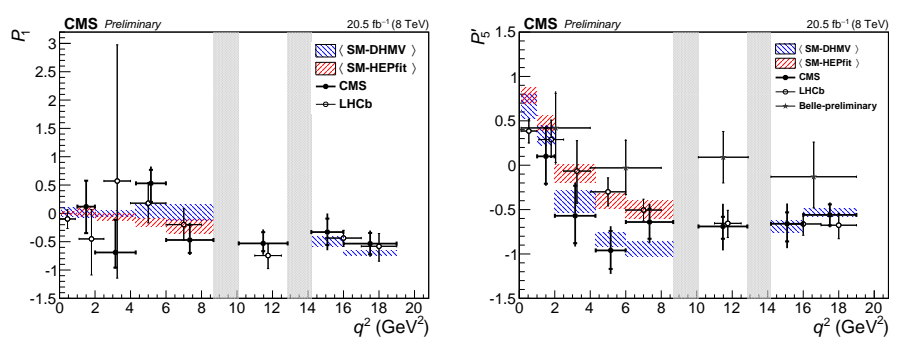

Figure 6: Measured values of $P_{1}$ (left) and $P_{5}^{\prime}$ (right) versus $q^{2}$ for $B^{0} \rightarrow K^{* 0} \mu^{+} \mu^{-}$[21], compared with results from LHCb [12] and Belle [22] as well as the SM predictions (blue) [20] and the HEP fit (red) [15].

CMS results seem in better agreement with Standard Model original predictions than the latest fit, anyway the analysis of new data will be necessary to clarify better the situation.

\section{Conclusions}

CMS has produced new results about $\mathrm{B}$ mesons and quarkonia production cross-sections; some previous measurements have been extended to $\sqrt{s}=13 \mathrm{TeV}$. An angular analysis of the decay $B^{0} \rightarrow K^{* 0} \mu^{+} \mu^{-}$has been performed, results have been compared to predictions and measurements from other experiments.

\section{References}

[1] CMS Collaboration, The CMS experiment at the CERN LHC JINST 03 (2008) S08004. 
[2] CMS Collaboration, Measurement of the total and differential inclusive $B^{+}$hadron cross sections in pp collisions at $\sqrt{s}=13 \mathrm{TeV}$ arXiv:1609.00873.

[3] CMS Collaboration, Measurement of the $B^{+}$Production Cross Section in pp Collisions at $\sqrt{s}=7 \mathrm{TeV}$ Phys. Rev. Lett. 106 (2011) 112001.

[4] A brief introduction to PYTHIA 8.1, T.Sjöstrand Comput. Phys. Commun. C 178 (2008) 852.

[5] M.Cacciari et al., Theoretical predictions for charm and bottom production at the LHC JHEP 1210 (2012) 137.

[6] M.Cacciari et al., Gluon PDF constraints from the ratio of forward heavy quark production at the LHC at $\sqrt{s}=7 \mathrm{TeV}$ and $\sqrt{s}=13 \mathrm{TeV}$ arXiv:1507.06197.

[7] P.Faccioli et al., Quarkonium production in the LHC era: A polarized perspective Phys. Lett. B 736 (2014) 98.

[8] G.T.Bodwin et al., Fragmentation Contributions to J/ $\psi$ Production at the Tevatron and the LHC Phys. Rev. Lett. 113 (2014) 022001.

[9] CMS Collaboration, Quarkonium production cross sections in pp collisions at $\sqrt{s}=13 \mathrm{TeV}$ CMS-PAS-BPH-15-005.

[10] CMS Collaboration, Measurement of $J / \psi$ and $\psi(2 S)$ Prompt Double-Differential Cross Sections in pp Collisions at $\sqrt{s}=7 \mathrm{TeV}$ Phys. Rev. Lett. 114 (2015) 191802.

[11] CMS Collaboration, Measurements of the $\Upsilon(1 S), \Upsilon(2 S)$ and $\Upsilon(3 S)$ differential cross sections in $p p$ collisions at $\sqrt{s}=7 \mathrm{TeV}$ Phys. Lett. B 749 (2015) 14.

[12] LHCb Collaboration, Angular analysis of the $B^{0} \rightarrow K^{* 0} \mu^{+} \mu^{-}$decay using $3 \mathrm{fb}^{-1}$ of integrated luminosity JHEP 1602 (2016) 104.

[13] S.Descotes-Genon et al., Global analysis of $b \rightarrow$ sll anomalies JHEP 1606 (2016) 092.

[14] M.Ciuchini et al., $B \rightarrow K^{*} \ell^{+} \ell^{-}$decays at large recoil in the Standard Model: a theoretical reappraisal JHEP 1606 (2016) 116.

[15] M.Ciuchini et al., $B \rightarrow K^{*} \ell^{+} \ell^{-}$in the Standard Model: Elaborations and Interpretations arXiv:1611.04338.

[16] CMS Collaboration, Angular analysis of the decay $B^{0} \rightarrow K^{* 0} \mu^{+} \mu^{-}$from pp collisions at $\sqrt{s}=8 \mathrm{TeV}$ Phys. Lett. B 753 (2016) 424.

[17] A.Khodjamirian et al., Charm-loop effect in $B \rightarrow K^{(*)} \ell^{+} \ell^{-}$and $B \rightarrow K^{(*)} \gamma$ JHEP 1009 (2010) 089.

[18] A.Khodjamirian et al., $B \rightarrow K \ell^{+} \ell^{-}$decay at large hadronic recoil JHEP 1302 (2013) 010.

[19] R.R.Horgan et al., Lattice QCD calculation of form factors describing the rare decays $B \rightarrow K^{*} \ell^{+} \ell^{-}$ and $B_{s} \rightarrow \phi \ell^{+} \ell^{-}$Phys. Rev. D 89 (2014) 094501.

[20] S.Descotes-Genon et al., Optimizing the basis of $B \rightarrow K^{*} \ell^{+} \ell^{-}$observables in the full kinematic range JHEP 1305 (2013) 137.

[21] CMS Collaboration, Measurement of the $P_{1}$ and $P_{5}^{\prime}$ angular parameters of the decay $B^{0} \rightarrow K^{* 0} \mu^{+} \mu^{-}$ in proton-proton collisions at $\sqrt{s}=8 \mathrm{TeV}$ CMS-PAS-BPH-15-008.

[22] Belle Collaboration, Lepton-Flavor-Dependent Angular Analysis of $B \rightarrow K^{*} \ell^{+} \ell^{-}$Phys. Rev. Lett. 118 (2017) 111801. 